\title{
COVID-19 Vaccine Acceptance In Pregnancy
}

Priya Desai, OSM IV., Gagandeep Kaur ,OSM IV., Fanglong Dong, PhD, Maria Hellen Rodriguez, MD

\begin{abstract}
:
Background: Early in the COVID-19 pandemic, limited data existed regarding the safety and efficacy of the COVID-19 vaccine in pregnant women. As such, many pregnant women have reservations about receiving the COVID-19 vaccine. However, pregnant women are of particular concern as they are considered a vulnerable population due to the increased risk of severe complications from COVID-19. This study investigates the willingness of pregnant patients to receive the vaccine before and after discussion with a health care provider.

Methods: 124 pregnant women were surveyed from February to March of 2021 at a perinatal center. Patients were queried about their willingness to receive the COVID-19 vaccine before and after reading a fact sheet and discussing the vaccine's safety with a provider.

Results: There was a statistically significant effect of discussing the vaccine with a physician regarding patients' willingness to receive the COVID-19 vaccine. Additionally, patients who received the annual influenza vaccine were significantly more likely to get the COVID-19 vaccine.

Conclusion: Pregnant women were more willing to receive the COVID-19 vaccine afterdiscussion with a health care provider. The importance and impact of health education should be given special consideration in medical offices.

\section{"While COVID-19 vaccines will soon become available to the general public, there remains uncertainty about the safety and efficacy of the COVID-19 vaccine in pregnant women amongst healthcare professionals."}

\section{Introduction:}

The COVID-19 pandemic has caused 2.7 million deaths worldwide, including more than 500,000 in the United States. While COVID-19 vaccines will soon become available to the general public, there remains uncertainty about the safety and efficacy of the COVID-19 vaccine in pregnant women amongst healthcare professionals.

Lactating and pregnant women were not included in the original phase 3 COVID-19 vaccine clinical trials. Therefore limited data exist regarding long-term outcomes in pregnancy. However, the COVID-19 infection is of particular concern in pregnancy. More than 73,000 infections and 80 deaths have been documented in pregnant women since the start of the pandemic in the United States. Infected pregnant individuals are at increased risk for severe illness, admission to the intensive care unit, mechanical ventilation, pregnancy complications, and death compared to nonpregnant counterparts. (1)

Given the vulnerability of this population, the American College of Obstetricians and Gynecologists and the CDC recommend the authorization and use of the COVID-19 vaccines in pregnant women. Several studies have analyzed the acceptance and willingness of the general population to receive the COVID-19 vaccine in the United States. However, there is limited data regarding the attitudes and perceptions of pregnant women towards receiving the vaccine. The decision to receive the vaccine is asource of confusion and conflict for many pregnant women, making this an important topic of discussion between patients and obstetrics and gynecology providers. (2-5)

\section{Objectives/Purpose:}

This study investigates pregnant patients' willingness to receive the COVID-19 vaccine before and after discussion with a health care provider.

Demographic factors associated with vaccine willingness were also studied.

\section{"An anonymous cross-sectional survey of pregnant women ages 18 years or older was conducted at Pomona Valley Hospital's perinatal center between February and March 2021. Patients were queried about their willingness to receive the COVID-19 vaccine before they were given any information. Data on demographic factors, including race, religion, education level, marital status, and prior vaccine history, were also collected."}

\section{Methodology:}

An anonymous cross-sectional survey of pregnant women ages 18 years or older was conducted at Pomona Valley Hospital's perinatal center between February and March 2021. Patients were queried about their willingness to receive the COVID-19 vaccine before they were given any information. Data on demographic factors, including race, religion, education level, marital status, and prior vaccine history, were also collected. Patients were then asked to read a fact sheet about the safety of the COVID-19 vaccines in pregnant women and discussed the information with a provider. A comparison between the pre and post willingness to receive the COVID-19 vaccine was conducted. A p-value $<0.05$ was considered to be statistically significant. The study received IRB approval from the Pomona Valley Hospital institutional review board.

\section{Results:}

A total of 124 patients were included in the final analysis, with an average age of $29.9(S D=2.89)$ years and an average gestational age of $31.2(S D=6.69)$ weeks. More than half $(58.9 \%, n=73)$ were 


\section{Influenza vaccine vs Covid19 vaccine}

$100 \%$

$80 \%$

$60 \% \quad 56.5 \%$
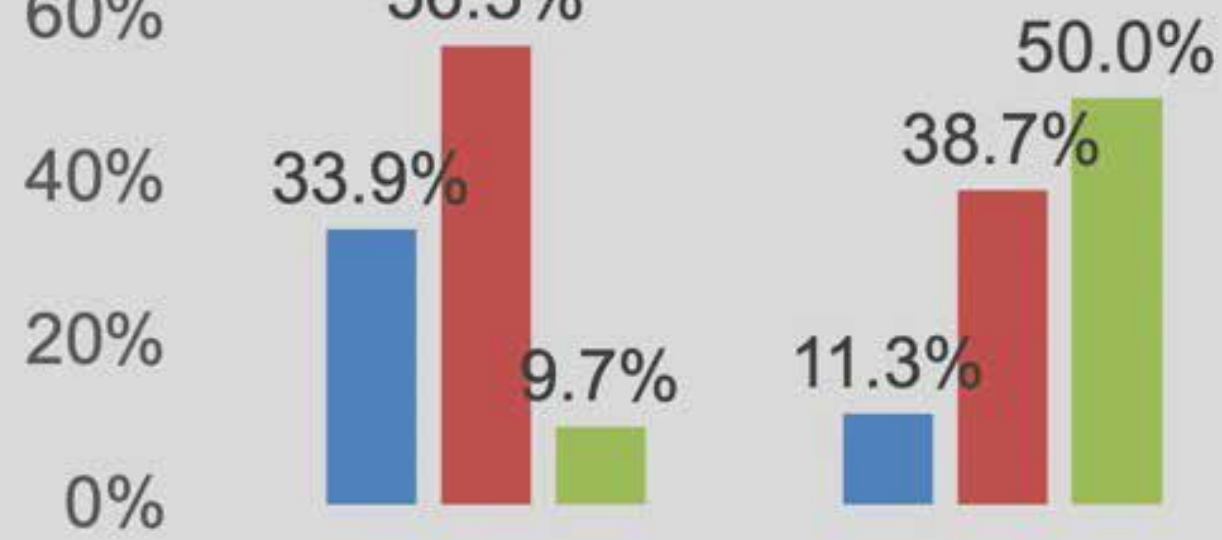

Not willing to get Covid19 vaccine

- Unsure about covid19 vaccine Wiling to receive Covid19 vaccine

\section{Did not receive influenza vaccine}

Received influenza vaccine

Hispanic, and almost half $(49.2 \%)$ had a highschool or lower degree. The majority $(89.1 \%, n=106)$ of participants were in their first trimester of pregnancy. Half $(50 \%, n=62)$ of the participants reported that they received influenza in the past, and more than half $(59.7 \%, n=74)$ reported receiving the Tdap vaccine. Those who received the annual influenza vaccine were significantly more likely to get the COVID-19 vaccine (50\% vs. 9.7\%, p<0.0001). Additionally, those who had previously discussed the COVID-19 vaccine with a physician were significantly more likely to receive the vaccine $(45.8 \%$ vs. $26.0 \%, p=0.0426)$. There was a statistically significant effect of discussing the vaccine with a healthcare provider and providing patients with a fact sheet regarding patients' willingness to receive the COVID-19 vaccine $(p<0.0001)$.

\section{Conclusions/Discussion:}

In this cross-sectional sample of patients, pregnant patients who discussed the COVID-19 vaccine with a healthcare provider were statistically more likely to express willingness to receive the vaccine. This study also found that higher education level and previous influenza vaccination are factors associated with vaccine willingness.

Although not statistically significant, women with an associate's degree or higherwere more willing to receive the COVID-19 vaccine when compared to women with a high school education or less. This result is consistent with a study discussed in Ceulemans et al. (6), which reported that pregnant and breastfeeding women with low to medium education levels were less likely to get the COVID-19 vaccine when it became available to them. Similarly, unemployment was associated with vaccine hesitancy. Fisher et al. (7) reported that anti-vaccine attitudes, lack of trust in the healthcare system and vaccine development, and the need for more information regarding the safety and efficacy of the vaccine were amongst the reasons for intending not to be vaccinated. Many other previous studies have similarly reported increased willingness to receive the COVID-19 vaccine among patients with higher education.

"In this cross-sectional sample of patients, pregnant patients who discussed the COVID-19 vaccine with a healthcare provider were statistically more likely to express willingness to receive the vaccine. This study also found that higher education level and previous influenza vaccination are factors associated with vaccine willingness."

Another independent factor that played a role in willingness to receive the COVID-19 vaccine was previous influenza and Tdap vaccination. Women who had received influenza and Tdap vaccination were more likely to receive the COVID-19 vaccine than those unvaccinated. According to Fisher et al. (7), evidence 


\section{$100 \%$}

\section{$80 \%$}

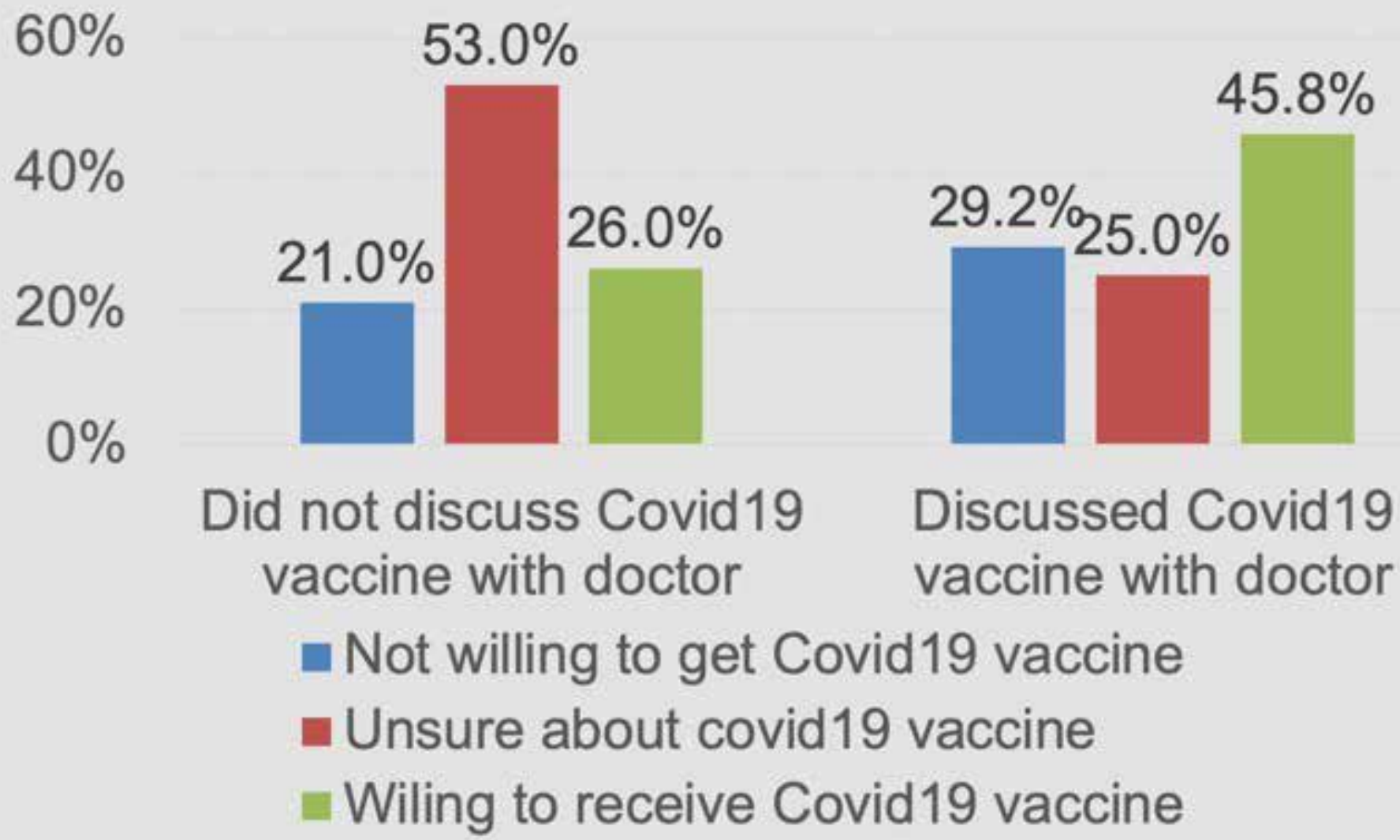

shows that patients whose physicians recommend a vaccine are more likely to be vaccinated than patients whose physicians do not. Prior influenza and Tdap vaccination may be reflective of attitudes toward and trust in vaccinations in general.

Pregnant women have historically been excluded from many vaccine trials. As a result, there is a lack of evidence supporting the safety and efficacy of vaccines in this specific population. Data on vaccine safety in pregnant women are often drawn from the limited number of inadvertent exposures during pregnancy. There is a critical need for the inclusion of pregnant women in vaccine trials, given the vulnerability of this population to developing severe illness with COVID-19. Vaccination of pregnant women can also decrease household transmission of COVID-19 among children too young to receive the vaccine (8). Greater evidence on vaccine safety amongst pregnant women can increase vaccine confidence and trust in both pregnant women and their obstetrics providers.

After reading the informational fact sheet and discussing it with a health care provider, more women were willing to receive the $\mathrm{CO}$ VID-19 vaccine. Similarly, the effect of educational intervention on vaccine acceptance was studied in China in 2019. (9) The attitude towards and willingness to receive the HPV vaccine was documented before and after an informative presentation. More students were willing to vaccinate themselves after health education. Policymakers should give efforts to educate vulnerable popula- tions more consideration in order to improve vaccine acceptance.

The potential limitations of this study include a sample restricted to 124 patients at a single perinatal center. Furthermore, several patients reported having already beenoffered the COVID-19 vaccine due to their employment status as essential workers.

Lastly, patients who had already discussed the COVID-19 with their primary care providers may have had access to safety information regarding the vaccine prior to reading the COVID-19 fact sheet from their obstetrics provider.

Future studies should investigate potential reasons for vaccine reluctance and effective methods to deliver information to patients to encourage vaccine acceptance. (9) Preliminary findings from the largest study conducted to date on mRNA COVID-19 vaccine safety in pregnant women currently indicate no obvious safety concerns for this population. (10) As the United States finds itself at a turning point in the COVID-19 pandemic, it is more important than ever to prioritize strategies to promote vaccine acceptance.

More pregnant women were willing to receive the COVID-19 vaccine after discussion with a healthcare provider. Prior influenza and Tdap vaccination and higher education level were factors associated with greater vaccine willingness. The importance and impact of regular health education should be given special consideration in the pregnant patient population. 
$40 \%$



$20 \%$

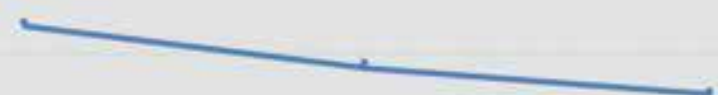

-Not willing to get Covid19 vaccine - Unsure about covid 19 vaccine -Wiling to receive Covid 19 vaccine

\section{pre factsheet Discuss \\ with \\ doctor}

\section{References:}

1. Bianchi DW, Kaeser L, Cernich AN. Involving Pregnant Individuals in Clinical Research on COVID-19 Vaccines. JAMA. 2021;325(11):1041-1042. doi:10.1001/jama.2021.1865

2. Riley, L. E., \& Jamieson, D. J. (2021). Inclusion of Pregnant and Lactating Persons in COVID-19 Vaccination Efforts. Annals of Internal Medicine. https://doi.org/10.7326/m21-0173

3. Mappa I, Luviso M, Distefano FA, Carbone L, Maruotti GM, Rizzo G. Women perception of SARS-CoV-2 vaccination during pregnancy and subsequent maternal anxiety: a prospectiveobservational study. J Matern Fetal Neonatal Med. 2021 Apr 11:1-4. doi: 10.1080/14767058.2021.1910672. Epub ahead of print. PMID: 33843419.

4. Gray KJ, Bordt EA, Atyeo C, Deriso E, Akinwunmi B, Young $N$, Medina Baez A, Shook LL,Cvrk D, James K, De Guzman $R$, Brigida S, Diouf K, Goldfarb I, Bebell LM, Yonker LM, Fasano A, Rabi SA, Elovitz MA, Alter G, Edlow AG, COVID-19 vaccine response in pregnant and lactating women: a cohort study, American Journal of Obstetrics and Gynecology (2021), doi: https://doi.org/10.1016/j.ajog.2021.03.023.

5. Szilagyi PG, Thomas K, Shah MD, et al. National Trends in the US Public's Likelihood of Getting a COVID-19 VaccineApril 1 to December 8, 2020. JAMA. 2021;325(4):396-398. doi:10.1001/jama.2020.26419

6. Ceulemans M, Foulon V, Panchaud A, Winterfeld U, Pomar $L$, Lambelet V, Cleary B, O'Shaughnessy F, Passier A, Rich- ardson JL, Allegaert K, Nordeng H. Vaccine Willingness and Impact of the COVID-19 Pandemic on Women's Perinatal Experiences and Practices-A Multinational, Cross-Sectional Study Covering the First Wave of the Pandemic. International Journal of Environmental Research and Public Health. 2021; 18(7):3367. https://doi.org/10.3390/ijerph18073367

7. Fisher, K. A., Bloomstone, S. J., Walder, J., Crawford, S., Fouayzi, H., \& Mazor, K. M. (2020). Attitudes Toward a Potential SARS-CoV-2 Vaccine. Annals of Internal Medicine, 173(12), 964-973. https://doi.org/10.7326/m20-3569

8. Beigi RH, Krubiner C, Jamieson DJ, et al. The need for inclusion of pregnant women in COVID-19 vaccine trials. Vaccine. 2021;39(6):868-870. doi:10.1016/j.vaccine.2020.12.074

9. Liu, C.-R., Liang, H., Zhang, X., Pu, C., Li, Q., Li, Q.-L., Li, J. (2019). Effect of an educational intervention on HPV knowledge and attitudes towards HPV and its vaccines among junior middle school students in Chengdu, China. BMC Public Health, 19(1). https://doi.org/10.1186/s12889-019-6823-0

10. Shimabukuro, T. T., Kim, S. Y., Myers, T. R., Moro, P. L., Oduyebo, T., Panagiotakopoulos, L., Meaney-Delman, D. M. (2021). Preliminary Findings of mRNA Covid-19 Vaccine Safety in Pregnant Persons. New England Journal of Medicine. https://doi.org/10.1056/nejmoa2104983 


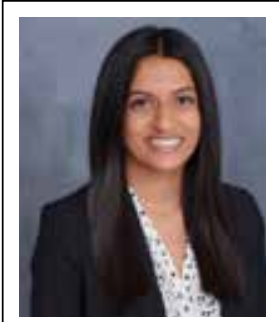

Priya Desai, OMS IV

Western University School of Medicine

Pomona, CA campus

309 E. Second St.

Pomona, CA 91766-1854

Corresponding Author

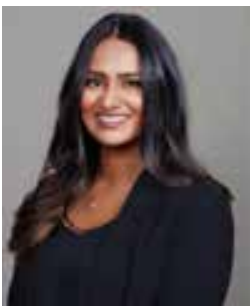

Gagandeep Kaur, OMS IV

Western University School of Medicine

Pomona, CA campus

309 E. Second St.

Pomona, CA 91766-1854

Email: Gagandeep Kaur <gagandeep.lal@westernu.edu>

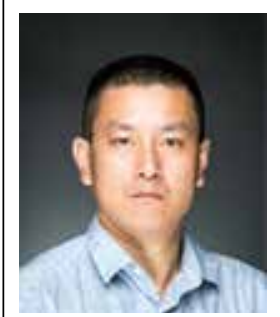

Fanglong Dong, PhD

Associate Professor

Graduate College of Biomedical Sciences

Western University of Health Sciences

309 E. Second St.

Pomona, CA 91766-1854

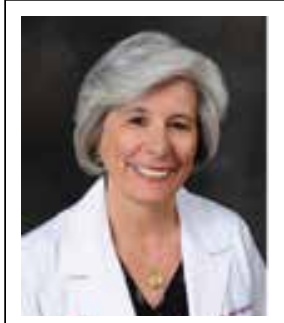

Maria Hellen Rodriguez, MD

Maternal and Fetal Medicine Physician

Pomona Valley Hospital

Pomona, CA

\section{Fellow's Column is published monthly.}

- Submission guidelines for "Fellow's Column":

- 2000 word limit not including references or title page. Exceptions will be made on a case by case basis

- $\quad$ QI/QA work, case studies, or a poster from a scientific meeting may be submitted..

- Submission should be from a medical student, resident, fellow, or NNP in training.

- Topics may include Perinatology, Neonatology, and Younger Pediatric patients.

- $\quad$ No more than 20 references.

- $\quad$ Please send your submissions to:

Elba Fayard, MD, Interim Fellowship Column Editor or Japmeet Sandhu, OMS III, Fellowship Column Assistant Editor LomaLindaPublishingCompany@gmail.com

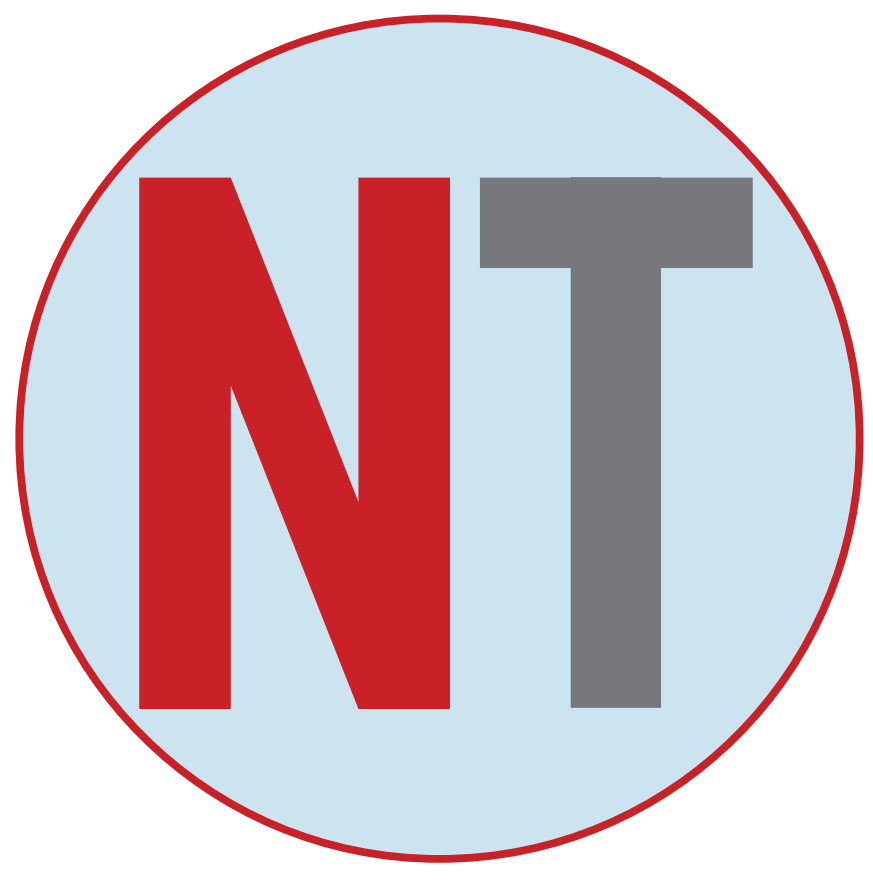

